Moroccan J. of Pure and Appl. Anal. (MJPAA)

Volume 6(2), 2020, Pages 281-302

ISSN: Online 2351-8227 - Print 2605-6364

DOI: $10.2478 / \mathrm{mjpaa}-2020-0022$

\title{
Some class of nonlinear inequalities with gradient constraints in Orlicz spaces
}

\author{
S. AJAgJal ${ }^{1}$ AND D. MesKine ${ }^{2}$
}

Aвstract. In the present paper, we show the existence of solutions of some nonlinear inequalities of the form $\langle A u+g(x, u, \nabla u), v-u\rangle \geq\langle f, v-u\rangle$ with gradient constraint that depend on the solution itself, where $A$ is a Leray-Lions operator defined on Orlicz spaces, $g$ is a nonlinearity and $f \in L^{1}$.

Mathematics Subject Classification (2020). 35J87, 35J60, 35J92, 46E30.

Key words and phrases. Nonlinear variational inequalities, Nonlinear elliptic equations, $p$-Laplacian, Orlicz spaces.

\section{Introduction}

Problems with gradient constraint have recently been the subject of many mathematical and numerical studies, also these kind of inequalities arising in different physical models, such that sandpile growth [12], the superconductivity see [13] and also in image processing [2].

In this paper we deal with some nonlinear inequalities with obstacles depending on the gradients of the solutions in the setting of Orlicz-Sobolev spaces.

Let $\Omega$ be a bounded subset of $\mathbb{R}^{N}(N \geq 2)$ with the segment property.

Let $A$ be a Leray-Lions operator defined on $D(A) \subset W_{0}^{1} L_{M}(\Omega) \rightarrow W^{-1} L_{\bar{M}}(\Omega)$, with $M$ an

Received : 15 June 2020 - Accepted: 30 September 2020.

(C) The author(s) 2020. This article is published with open access by Sidi Mohamed Ben Abdallah University.

1,2Ecole Supérieure de Technologie d'Essaouira, B.P. 383 Essaouira El Jadida, Essaouira.

e-mail: ${ }^{1}$ sana.ajagjal@gmail.com

e-mail:2dr.meskine@uca.ac.ma (Corresponding author). 
$N$-function and consider the non-linearity $g$ which satisfies the following "natural" growth condition:

$$
|g(x, s, \xi)| \leq b(|s|)(c(x)+M(|\xi|))
$$

and the classical sign condition $g(x, s, \xi) s \geq 0$.

Our goal is to prove the existence result of the solution of the following problem

$$
(\mathcal{P})\left\{\begin{array}{l}
\langle A u+g(x, u, \nabla u), v-u\rangle \geq\langle f, v-u\rangle, \quad \forall v \in K_{u} \\
u \in K_{u},
\end{array}\right.
$$

where $K_{u}$ is the convex defined as follows

$$
K_{u}=\left\{v \in W_{0}^{1, \infty}(\Omega):|\nabla v| \leq h(u) \text { a.e. in } \Omega\right\}
$$

where $h$ is a nonnegative continuous function such that $\int_{0}^{+\infty} \frac{d s}{h(s)}=+\infty$ and $h \geq \delta$ with $\delta>0$ is a real number and $f \in L^{1}(\Omega), f \geq 0$.

In order to get our aim, we deal with the asymptotic behaviour of the entropy solution $u_{p}$ $(p>1)$ of the following problem, when $p$ goes to $\infty$

$$
\begin{cases}-\operatorname{div}\left(\frac{\left|\nabla u_{p}\right|^{p-2}}{h\left(u_{p}\right)^{p-1}} \nabla u_{p}\right)-\operatorname{div}\left(a\left(x, u_{p}, \nabla u_{p}\right)\right)+g\left(x, u_{p}, \nabla u_{p}\right)=f & \text { in } \Omega \\ u_{p}=0 & \text { on } \partial \Omega\end{cases}
$$

where $f \in L^{1}(\Omega)$ and $h$ is the continuous function defined above and which can be for example $h(s)=1+|s|$ or $h(s)=1+|s| \log ^{\alpha}(e+|s|)$ with $\alpha \leq 1$.

The existence of the entropy solution of this problem has been proved by A. Elmahi and D. Meskine in [4], they also solved it in the variational case (see [5]).

Firstly, we will study the limit when $p$ goes to infinity of $u_{p}$ and we will show that, up to a subsequence, we have

$$
u_{p} \rightarrow u \text { a.e. in } \Omega \text {. }
$$

Moreover, we show that $u$ satisfies

$$
|\nabla u| \leq h(u) \text { a.e. in } \Omega \text {. }
$$

Secondly, we will prove that this function limit $u$ is solution of the obstacle problem $(\mathcal{P})$.

The plan of this paper is as follows: in section 2 we recall some preliminaries concerning Orlicz-Sobolev spaces and some lemmas applied on the truncation operators while in section 3 we prove the existence of solutions for problems of the forme $(\mathcal{P})$.

\section{Notations and Preliminaries}

In this section we recall some notations and preliminaries that will be used later. First, we start with recalling the definition of N-Functions, Orlicz spaces and Orlicz-Sobolev spaces. 


\section{$N$-Functions.}

Let $M: \mathbb{R}^{+} \rightarrow \mathbb{R}^{+}$be an $N$-function, i.e. $M$ is continuous, convex, with $M(t)>0$ for $t>0$, $\frac{M(t)}{t} \rightarrow 0$ as $t \rightarrow 0$ and $\frac{M(t)}{t} \rightarrow \infty$ as $t \rightarrow \infty$.

Equivalently, $M$ admits the representation: $M(t)=\int_{0}^{t} a(s) d s$, where $a: \mathbb{R}^{+} \rightarrow \mathbb{R}^{+}$is nondecreasing, right continuous, with $a(0)=0, a(t)>0$ for $t>0$ and $a(t)$ tends to $\infty$ as $t \rightarrow \infty$.

The $N$-function $\bar{M}$ conjugate to $M$ is defined by $\bar{M}(t)=\int_{0}^{t} \bar{a}(s) d s$, where $\bar{a}: \mathbb{R}^{+} \rightarrow \mathbb{R}^{+}$is given by $\bar{a}(t)=\sup \{s: a(s) \leq t\}$ (see [1], [10],[9]).

While the $N$-function $M$ satisfy for some $k>0$,

$$
M(2 t) \leq k M(t) \quad \forall t \geq 0,
$$

we said that satisfy the $\triangle_{2}$-condition, when (2.1) holds only for $t \geq$ some $t_{0}>0$, then $M$ is said to satisfy the $\triangle_{2}$-condition near infinity.

Let $P$ and $H$ two $\mathrm{N}$-functions, $P \ll H$ means that $P$ grows less rapid to $H$, i.e. for all $\eta>0$

$$
\frac{P(t)}{H(\eta t)} \rightarrow 0 \text { as } t \rightarrow \infty
$$

\section{Orlicz spaces.}

Let $\Omega$ be an open subset of $\mathbb{R}^{N}$. The Orlicz class $K_{M}(\Omega)$ (resp. the Orlicz space $L_{M}(\Omega)$ ) is defined as the set of (equivalence classes of) real valued measurable functions $u$ on $\Omega$ such that:

$$
\int_{\Omega} M(u(x)) d x<+\infty\left(\text { resp. } \int_{\Omega} M\left(\frac{u(x)}{\lambda}\right) d x<+\infty \text { for some } \lambda>0\right) .
$$

$L_{M}(\Omega)$ is a Banach space under the norm

$$
\|u\|_{M, \Omega}=\inf \left\{\lambda>0: \int_{\Omega} M\left(\frac{u(x)}{\lambda}\right) d x \leq 1\right\}
$$

and $K_{M}(\Omega)$ is a convex subset of $L_{M}(\Omega)$.

The closure in $L_{M}(\Omega)$ of the set of bounded measurable functions with compact support in $\bar{\Omega}$ is denoted by $E_{M}(\Omega)$.

The equality $E_{M}(\Omega)=L_{M}(\Omega)$ holds if and only if $M$ satisfies the $\triangle_{2}$-condition for all $t$ or for $t$ large according to whether $\Omega$ has infinite measure or not.

The dual of $E_{M}(\Omega)$ can be identified with $L_{\bar{M}}(\Omega)$ by means of the pairing $\int_{\Omega} u(x) v(x) d x$, and the dual norm on $L_{\bar{M}}(\Omega)$ is equivalent to $\|\cdot\|_{\bar{M}}$.

The space $L_{M}(\Omega)$ is reflexive if and only if $M$ and $\bar{M}$ satisfy the $\triangle_{2}$-condition, for all $t$ or for $t$ large, according to whether $\Omega$ has infinite measure or not.

Orlicz-Sobolev spaces.

The space of all functions $u$ such that $u$ and its distributional derivatives up to order 1 lie in $L_{M}(\Omega)\left(\right.$ resp. $\left.E_{M}(\Omega)\right)$ is denoted by $W^{1} L_{M}(\Omega)$ (resp. $W^{1} E_{M}(\Omega)$ ). It is a Banach space under 
the norm

$$
\|u\|_{1, M}=\sum_{\alpha \leq 1}\left\|D^{\alpha} u\right\|_{M}
$$

Thus, $W^{1} L_{M}(\Omega)$ and $W^{1} E_{M}(\Omega)$ can be identified with subspaces of product of $N+1$ copies of $L_{M}(\Omega)$. Denoting this product by $\prod L_{M}$, we will use the weak topologies $\sigma\left(\prod L_{M}, \prod E_{\bar{M}}\right)$ and $\sigma\left(\prod L_{M}, \prod L_{\bar{M}}\right)$.

The space $W_{0}^{1} E_{M}(\Omega)$ is defined as the (norm) closure of the Schwarz space $D(\Omega)$ in $W^{1} E_{M}(\Omega)$ and the space $W_{0}^{1} L_{M}(\Omega)$ as the $\sigma\left(\prod L_{M}, \prod E_{\bar{M}}\right)$ closure of $D(\Omega)$ in $W^{1} L_{M}(\Omega)$.

We say that $u_{n}$ converges to $u$ for the modular convergence in $W^{1} L_{M}(\Omega)$ if for some $\lambda>0$

$$
\int_{\Omega} M\left(\frac{D^{\alpha} u_{n}-D^{\alpha} u}{\lambda}\right) d x \rightarrow 0 \text { for all } \alpha \leq 1 \text {. }
$$

This implies convergence for $\sigma\left(\prod L_{M}, \prod L_{\bar{M}}\right)$.

If $M$ satisfies the $\triangle_{2}$-condition on $\mathbb{R}^{+}$(near infinity only if $\Omega$ has finite measure), then modular convergence coincides with norm convergence.

Let $W^{-1} L_{\bar{M}}(\Omega)$ ( resp. $W^{-1} E_{\bar{M}}(\Omega)$ ) denote the space of distribution on $\Omega$ which can written as sums of derivatives of order $\leq 1$ of functions in $L_{\bar{M}}(\Omega)$ ( resp. $E_{\bar{M}}(\Omega)$ ). It is a Banach space under the usual quotient norm.

If the open set $\Omega$ has the segment property then the space $D(\Omega)$ is dense in $W_{0}^{1} L_{M}(\Omega)$ for the modular convergence and thus for the topology $\sigma\left(\prod L_{M}, \prod L_{\bar{M}}\right)$ (see [6], [7]). Consequently, the action of a distribution $S$ in $W^{-1} L_{\bar{M}}(\Omega)$ on an element $u$ of $W_{0}^{1} L_{M}(\Omega)$ is well defined. It will be denoted by $\langle S, u\rangle$.

Now, we quote some lemmas applied on to the truncation operators.

Lemma 1. Let $F: \mathbb{R} \longrightarrow \mathbb{R}$ be uniformly Lipschitzian, with $F(0)=0$. Let $M$ be an $N$-function and let $u \in W^{1} L_{M}(\Omega)\left(\operatorname{resp} . W^{1} E_{M}(\Omega)\right)$. Then $F(u) \in W^{1} L_{M}(\Omega)$ ( resp. $\left.W^{1} E_{M}(\Omega)\right)$. Moreover, if the set $D$ of discontinuity points of $F^{\prime}$ is finite, we have

$$
\frac{\partial}{\partial x_{i}} F(u)=\left\{\begin{array}{ccc}
F^{\prime}(u) \frac{\partial u}{\partial x_{i}} & \text { a.e in } & \{x \in \Omega: u(x) \notin D\}, \\
0 & \text { a.e in } & \{x \in \Omega: u(x) \in D\} .
\end{array}\right.
$$

Lemma 2. Let $F: \mathbb{R} \longrightarrow \mathbb{R}$ be uniformly Lipschitzian, with $F(0)=0$. We suppose that the set of discontinuity points of $F^{\prime}$ is finite. Let $M$ be an N-function, then the mapping: $F: W^{1} L_{M}(\Omega) \rightarrow$ $W^{1} L_{M}(\Omega)$ is sequentially continuous with respect to the weak topology $\sigma\left(\prod L_{M}, \prod E_{\bar{M}}\right)$.

Lemma 3. Let $\Omega$ be an open subset of $\mathbb{R}^{N}$ with finite measure. Let $M, P$ and $Q$ be $N$-functions such that $Q \ll P$, and let $f: \Omega \times \mathbb{R} \rightarrow \mathbb{R}$ be a Carathéodory function such that, for a.e. $x \in \Omega$ and for all $s \in \mathbb{R}$,

$$
|f(x, s)| \leq c(x)+k_{1} P^{-1} M\left(k_{2} s\right),
$$

where $k_{1}, k_{2}$ are real constants and $c(x) \in E_{Q}(\Omega)$. Then the Nemytskii operator $N_{f}$ defined by $N_{f}(u)(x)=f(x, u(x))$, is strongly continuous from

$$
\mathcal{P}\left(E_{M}(\Omega), \frac{1}{K_{2}}\right)=\left\{u \in L_{M}(\Omega): d\left(u, E_{M}(\Omega)\right)<\frac{1}{k_{2}}\right\}
$$


into $E_{Q}(\Omega)$.

Lemma 4. Let $\left(f_{n}\right), f \subset L^{1}(\Omega)$ such that

(1) $f_{n} \geq 0$ a.e in $\Omega$;

(2) $f_{n} \rightarrow f$ a.e in $\Omega$;

(3) $\int_{\Omega} f_{n}(x) d x \rightarrow \int_{\Omega} f(x) d x$;

then $f_{n} \rightarrow f$ strongly in $L^{1}(\Omega)$.

\section{The existence result}

Let $\Omega$ be a bounded open subset of $\mathbb{R}^{N}$ with the segment property.

Let $M$ and $P$ be two $N$-functions such that $P \ll M$.

Let $A: D(A) \subset W_{0}^{1} L_{M}(\Omega) \longrightarrow W^{-1} L_{\bar{M}}(\Omega)$ be a mapping (non-everywhere defined) given by

$$
A\left(u_{p}\right)=-\operatorname{div}\left(a\left(x, u_{p}, \nabla u_{p}\right)\right) \text {, }
$$

for all $p>1$, where $a: \Omega \times \mathbb{R} \times \mathbb{R}^{N} \longrightarrow \mathbb{R}^{N}$ is a Carathéodory function satisfying, for a.e $x \in \Omega$, and for all $s \in \mathbb{R}$ and all $\xi, \xi^{*} \in \mathbb{R}^{N}, \xi \neq \xi^{*}$,

$$
\begin{gathered}
|a(x, s, \xi)| \leq c(x)+k_{1} \bar{P}^{-1} M\left(k_{2} s\right)+k_{3} \bar{M}^{-1} M\left(k_{4} \xi\right), \\
{\left[a(x, s, \xi)-a\left(x, s, \xi^{*}\right)\right]\left[\xi-\xi^{*}\right]>0,} \\
\alpha M(|\xi|) \leq a(x, s, \xi) \xi,
\end{gathered}
$$

where $c(x)$ belongs to $E_{\bar{M}}(\Omega), c \geq 0, k_{1}, k_{2}, k_{3}, k_{4}$ to $\mathbb{R}^{+}$and $\alpha \in \mathbb{R}_{*}^{+}$.

Let $h$ be a nonnegative continuous function such that

$$
\int_{0}^{+\infty} \frac{d s}{h(s)}=+\infty \text { and } h \geq \delta
$$

with $\delta>0$ is a real number.

Let $g(x, s, \xi): \Omega \times \mathbb{R} \times \mathbb{R}^{N} \rightarrow \mathbb{R}$ be a Carathéodory function such that for a.e. $x \in \Omega$ and for all $s \in \mathbb{R}, \xi \in \mathbb{R}^{N}$,

$$
\begin{gathered}
g(x, s, \xi) s \geq 0, \\
|g(x, s, \xi)| \leq b(|s|)\left(c^{\prime}(x)+M(|\xi|)\right),
\end{gathered}
$$

where $b: \mathbb{R} \rightarrow \mathbb{R}$ is a continuous and nondecreasing function and $c^{\prime}(x)$ is a given non-negative function in $L^{1}(\Omega)$.

Finally, we suppose that

$$
f \in L^{1}(\Omega), f \geq 0 .
$$

Consider the following elliptic problem with Dirichlet boundary condition:

$$
A\left(u_{p}\right)-\operatorname{div}\left(\frac{\left|\nabla u_{p}\right|^{p-2}}{h\left(u_{p}\right)^{p-1}} \nabla u_{p}\right)+g\left(x, u_{p}, \nabla u_{p}\right)=f \text { in } \Omega .
$$


Define $T_{0}^{1, M_{p}}(\Omega)$ to be the set of measurable functions $u: \Omega \rightarrow \mathbb{R}$ such that $T_{k}\left(u_{p}\right) \in$ $W_{0}^{1} L_{M_{p}}(\Omega)$, where $M_{p}(t)=M(t)+t^{p}, p>1$, and for all $k \geq 0$,

$$
T_{k}(s)=\max (-k, \min (k, s)) \text { for all } s \in \mathbb{R} .
$$

The existence of the solution of the problem (3.8) has been proved in [4] within the meaning of the following definition.

Definition 3.1. (Entropy solution) A function $u_{p}$ is an entropy solution of the equation (3.8) if:

$$
\left\{\begin{array}{l}
u_{p} \in T_{0}^{1, M_{p}}(\Omega), g\left(x, u_{p}, \nabla u_{p}\right) \in L^{1}(\Omega), \\
\left\langle A\left(u_{p}\right), T_{k}\left(u_{p}-v\right)\right\rangle+\int_{\Omega} \frac{\left|\nabla u_{p}\right|^{p-2}}{h\left(u_{p}\right)^{p-1}} \nabla u_{p} \nabla T_{k}\left(u_{p}-v\right) d x+\int_{\Omega} g\left(x, u_{p}, \nabla u_{p}\right) T_{k}\left(u_{p}-v\right) d x \\
\quad \leq \int_{\Omega} f T_{k}\left(u_{p}-v\right) d x \\
\text { for all } v \in W_{0}^{1} L_{M_{p}}(\Omega) \cap L^{\infty}(\Omega) \text { and for all } k \geq 0 .
\end{array}\right.
$$

The main result in this work is given by the following theorem:

Theorem 3.1. Assume that (3.1), (3.2), (3.3), (3.4), (3.5), (3.6) and (3.7) hold true. Let $u_{p}$ be an entropy solution of the problem (3.8), then there exists a measurable function $u$ such that, up to a subsequence, as $p$ goes to $\infty$,

in addition, we have

$$
u_{p} \rightarrow u \text { a.e in } \Omega,
$$

$$
|\nabla u| \leq h(u) \text { a.e in } \Omega \text {. }
$$

Moreover, $u$ satisfies the following inequality:

$$
\int_{\Omega} a(x, u, \nabla u)[\nabla u-\nabla v] d x+\int_{\Omega} g(x, u, \nabla u)(u-v) d x \leq\langle f, u-v\rangle,
$$

for all $v \in W_{0}^{1, \infty}(\Omega) \cap L^{\infty}(\Omega)$ such that $|\nabla v| \leq h(u)$.

Remark 3.1. Our result seems to be new even in the classical Sobolev spaces (see [11] -[13]).

Proof. In order to get this result, we start at first by establishing some a priori estimates. For every fixed $k>0$, taking $v=0$ in (3.9), so we have

$$
\begin{aligned}
\int_{\Omega} a\left(x, u_{p}, \nabla u_{p}\right) \nabla T_{k}\left(u_{p}\right) d x & +\int_{\Omega} \frac{\left|\nabla u_{p}\right|^{p-2}}{h\left(u_{p}\right)^{p-1}} \nabla u_{p} \nabla T_{k}\left(u_{p}\right) d x \\
& +\int_{\Omega} g\left(x, u_{p}, \nabla u_{p}\right) T_{k}\left(u_{p}\right) d x \leq \int_{\Omega} f T_{k}\left(u_{p}\right) d x
\end{aligned}
$$

since

$$
\int_{\left\{\left|u_{p}\right| \leq k\right\}} g\left(x, u_{p}, \nabla u_{p}\right) T_{k}\left(u_{p}\right) d x \geq 0,
$$


and

hence

$$
\int_{\left\{\left|u_{p}\right| \leq k\right\}} \frac{\left|\nabla u_{p}\right|^{p-2}}{h\left(u_{p}\right)^{p-1}} \nabla u_{p} \nabla T_{k}\left(u_{p}\right) d x \geq 0
$$

$$
\int_{\left\{\left|u_{p}\right| \leq k\right\}} a\left(x, u_{p}, \nabla u_{p}\right) \nabla u_{p} d x \leq C_{1} k
$$

where $C_{1}$ is a constant such that $\|f\|_{L^{1}(\Omega)} \leq C_{1}$. Therefore, by (3.3), we get

$$
\alpha \int_{\Omega} M\left(\left|\nabla T_{k}\left(u_{p}\right)\right|\right) d x \leq C_{1} k
$$

Thanks to Lemma 5.7 of [6], there exists two positive constants $\gamma$ and $\lambda$ such that

$$
\int_{\Omega} M(v) d x \leq \gamma \int_{\Omega} M(\lambda|\nabla v|) d x \text { for all } v \in W_{0}^{1} L_{M}(\Omega) .
$$

Taking $v=\frac{1}{\lambda} T_{k}\left(u_{p}\right)$, so we obtain

$$
\alpha \int_{\Omega} M\left(\frac{1}{\lambda} T_{k}\left(u_{p}\right)\right) d x \leq C_{2} k
$$

which implies that

For any $\delta>0$, we have

$$
\operatorname{meas}\left\{\left|u_{p}\right|>k\right\} \leq \frac{C_{3} k}{M\left(\frac{k}{\lambda}\right)}, \forall p>1, \forall k>0
$$

$$
\begin{aligned}
\text { meas }\left\{\left|u_{p}-u_{q}\right|>\delta\right\} \leq & \text { meas }\left\{\left|u_{p}\right|>k\right\}+\text { meas }\left\{\left|u_{q}\right|>k\right\} \\
& + \text { meas }\left\{\left|T_{k}\left(u_{p}\right)-T_{k}\left(u_{q}\right)\right|>\delta\right\}
\end{aligned}
$$

and so that

$$
\text { meas }\left\{\left|u_{p}-u_{q}\right|>\delta\right\} \leq \frac{2 C_{3} k}{M\left(\frac{k}{\lambda}\right)}+\text { meas }\left\{\left|T_{k}\left(u_{p}\right)-T_{k}\left(u_{q}\right)\right|>\delta\right\} \text {. }
$$

By using (3.11), we deduce that $\left(T_{k}\left(u_{p}\right)\right)_{p}$ is bounded in $W_{0}^{1} L_{M}(\Omega)$, and then there exists some $w_{k} \in W_{0}^{1} L_{M}(\Omega)$ such that

$$
\begin{array}{cl}
T_{k}\left(u_{p}\right) \rightarrow w_{k} & \text { weakly in } W_{0}^{1} L_{M}(\Omega) \text { for } \sigma\left(\Pi L_{M}, \Pi E_{\bar{M}}\right) \\
& \text { strongly in } E_{M}(\Omega) \text { and a.e in } \Omega .
\end{array}
$$

Consequently, we can assume that $\left(T_{k}\left(u_{p}\right)\right)$ is a Cauchy sequence in measure in $\Omega$.

Let $\epsilon>0$, there exists some $k(\epsilon)>0$ such that

$$
\text { meas }\left\{\left|u_{p}-u_{q}\right|>\delta\right\} \leq \epsilon, \text { for all } p, q \geq p_{0}(k(\epsilon), \delta) \text {. }
$$

This proves that $\left(u_{p}\right)$ is a Cauchy sequence in measure, thus, $u_{p}$ converges almost everywhere to some measurable function $u$.

By Lemma 4.4 [6], we obtain for all $k>0$,

$T_{k}\left(u_{p}\right) \rightarrow T_{k}(u)$ weakly in $W_{0}^{1} L_{M}(\Omega)$ for $\sigma\left(\Pi L_{M}, \Pi E_{\bar{M}}\right)$ strongly in $E_{M}(\Omega)$ and a.e in $\Omega$. 
On the other hand, thanks to (3.10) we get the following estimate

$$
\int_{\left\{\left|u_{p}\right| \leq k\right\}} \frac{\left|\nabla u_{p}\right|^{p}}{h\left(u_{p}\right)^{p-1}} d x \leq C_{1} k
$$

then

so

$$
\int_{\left\{\left|u_{p}\right| \leq k\right\}} \frac{\left|\nabla u_{p}\right|^{p}}{h\left(u_{p}\right)^{p}} h\left(u_{p}\right) d x \leq C_{1} k
$$

For every $q<p$, we have

$$
\int_{\left\{\left|u_{p}\right| \leq k\right\}} \frac{\left|\nabla u_{p}\right|^{p}}{h\left(u_{p}\right)^{p}} d x \leq C k
$$

$$
\int_{\Omega} \frac{\left|\nabla T_{k}\left(u_{p}\right)\right|^{q}}{h\left(u_{p}\right)^{q}} d x \leq\left(\int_{\Omega} \frac{\left|\nabla T_{k}\left(u_{p}\right)\right|^{p}}{h\left(u_{p}\right)^{p}} d x\right)^{\frac{q}{p}}|\Omega|^{1-\frac{q}{p}} \leq(C k)^{\frac{q}{p}}|\Omega|^{1-\frac{q}{p}}
$$

then

$$
\left(\int_{\Omega} \frac{\left|\nabla T_{k}\left(u_{p}\right)\right|^{q}}{h\left(u_{p}\right)^{q}} d x\right)^{\frac{1}{q}} \leq(C k)^{\frac{1}{p}}|\Omega|^{\frac{1}{q}-\frac{1}{p}}
$$

hence

$$
\left(\int_{\Omega}\left|\nabla\left(\int_{0}^{T_{k}\left(u_{p}\right)} \frac{d s}{h(s)}\right)\right|^{q}\right)^{\frac{1}{q}} \leq(C k)^{\frac{1}{p}}|\Omega|^{\frac{1}{q}-\frac{1}{p}} .
$$

Since $(C k)^{\frac{1}{p}}|\Omega|^{\frac{1}{q}-\frac{1}{p}} \rightarrow|\Omega|^{\frac{1}{q}}$ as $p \rightarrow+\infty$, then $\int_{0}^{T_{k}\left(u_{p}\right)} \frac{d s}{h(s)}$ is bounded in $W_{0}^{1, q}(\Omega)$ and since $T_{k}\left(u_{p}\right) \rightarrow T_{k}(u)$ a.e. in $\Omega$. So

$$
\int_{0}^{T_{k}\left(u_{p}\right)} \frac{d s}{h(s)} \rightarrow \int_{0}^{T_{k}(u)} \frac{d s}{h(s)} \text { in } W_{0}^{1, q}(\Omega) .
$$

Consequently, we have

$$
\left(\int_{\Omega}\left(\frac{\left|\nabla T_{k}(u)\right|}{h(u)}\right)^{q} d x\right)^{\frac{1}{q}} \leq|\Omega|^{\frac{1}{q}} .
$$

As the above inequality holds for every $q$, taking the limit as $q \rightarrow+\infty$, we get that

$$
\left|\nabla T_{k}(u)\right| \leq h\left(T_{k}(u)\right) \text { a.e. } x \in \Omega \text {, for all } k>0 .
$$

Now we will show that $u$ is bounded, indeed we have for $q_{0}>N$,

$$
\int_{\Omega}\left|\nabla\left(\int_{0}^{T_{k}(u)} \frac{d s}{h(s)}\right)\right|^{q_{0}} d x \leq|\Omega| .
$$

Then by Sobolev injection $W_{0}^{1, q_{0}}(\Omega)$ in $L^{\infty}(\Omega)$ we obtain

$$
\left|\int_{0}^{T_{k}(u)} \frac{d s}{h(s)}\right| \leq C .
$$


By letting $k \rightarrow+\infty$, we get

$$
\left|\int_{0}^{u} \frac{d s}{h(s)}\right| \leq C
$$

and since $\int_{0}^{+\infty} \frac{d s}{h(s)}=+\infty$, we conclude that

$$
|u| \leq M_{0} \quad \text { a.e. } \quad x \in \Omega \quad\left(M_{0} \text { is a constant }\right) .
$$

Go back to equation (3.12) and letting $k \rightarrow+\infty$, we obtain that

$$
|\nabla u| \leq h(u) \text { a.e. } x \in \Omega \text {. }
$$

Finally, since $\|u\|_{\infty} \leq k$, we get that

$T_{k}\left(u_{p}\right) \rightarrow u$ weakly in $W_{0}^{1} L_{M}(\Omega)$ for $\sigma\left(\Pi L_{M}, \Pi E_{\bar{M}}\right)$, and also weakly in $W_{0}^{1, q}(\Omega)(q>N)$

$$
\text { strongly in } E_{M}(\Omega) \text { and a.e in } \Omega \text {. }
$$

Now we will show that $\left(a\left(x, T_{k}\left(u_{p}\right), \nabla T_{k}\left(u_{p}\right)\right)\right)_{p}$ is bounded in $\left(L_{\bar{M}}(\Omega)\right)^{N}$ for all $k>0$, by using the dual norm of $\left(E_{\bar{M}}(\Omega)\right)^{N}$.

Let $\varphi \in\left(E_{\bar{M}}(\Omega)\right)^{N}$, such that $\|\varphi\|_{M, \Omega}=1$. In view of (3.2), we have

$$
\int_{\Omega}\left[a\left(x, T_{k}\left(u_{p}\right), \nabla T_{k}\left(u_{p}\right)\right)-a\left(x, T_{k}\left(u_{p}\right), \frac{\varphi}{k_{4}}\right)\right]\left[\nabla T_{k}\left(u_{p}\right)-\frac{\varphi}{k_{4}}\right] d x \geq 0,
$$

where $k_{i}(i=1, \ldots, 4)$ are the constants in (3.1).

This implies that

$$
\begin{aligned}
& \int_{\Omega} \frac{1}{k_{4}} a\left(x, T_{k}\left(u_{p}\right), \nabla T_{k}\left(u_{p}\right)\right) \varphi d x \\
& \quad \leq \int_{\Omega} a\left(x, T_{k}\left(u_{p}\right), \nabla T_{k}\left(u_{p}\right)\right) \nabla T_{k}\left(u_{p}\right) d x-\int_{\Omega} a\left(x, T_{k}\left(u_{p}\right), \frac{\varphi}{k_{4}}\right)\left[\nabla T_{k}\left(u_{p}\right)-\frac{\varphi}{k_{4}}\right] d x \\
& \quad \leq C_{1} k-\int_{\Omega} a\left(x, T_{k}\left(u_{p}\right), \frac{\varphi}{k_{4}}\right) \nabla T_{k}\left(u_{p}\right) d x+\frac{1}{k_{4}} \int_{\Omega} a\left(x, T_{k}\left(u_{p}\right), \frac{\varphi}{k_{4}}\right) \varphi d x,
\end{aligned}
$$

so, by using Young's inequality in the last two terms of last side of the above inequality, we deduce that

$$
\begin{aligned}
\int_{\Omega} a\left(x, T_{k}\left(u_{p}\right),\right. & \left.\nabla T_{k}\left(u_{p}\right)\right) \varphi d x \\
& \leq 3 k_{3}\left(1+k_{4}\right) \int_{\Omega} \bar{M}\left[\frac{a\left(x, T_{k}\left(u_{p}\right), \frac{\varphi}{k_{4}}\right)}{3 k_{3}}\right] d x \\
& +C_{1} k_{4} k+3 k_{3} k_{4} \int_{\Omega} M\left[\left|\nabla T_{k}\left(u_{p}\right)\right|\right] d x+3 k_{3} \int_{\Omega} M[\varphi] d x
\end{aligned}
$$




$$
\begin{aligned}
& \leq 3 k_{3}\left(1+k_{4}\right) \int_{\Omega} \bar{M}\left[\frac{\left|a\left(x, T_{k}\left(u_{p}\right), \frac{\varphi}{k_{4}}\right)\right|}{3 k_{3}}\right] d x \\
& +C_{1} k_{4} k+\frac{3 C_{1} k_{3} k_{4} k}{\alpha}+3 k_{3} .
\end{aligned}
$$

Moreover, thanks to (3.1) and the convexity of de $N$-function $\bar{M}$, we have

$$
\bar{M}\left[\frac{a\left(x, T_{k}\left(u_{p}\right), \frac{\varphi}{k_{4}}\right)}{3 k_{3}}\right] \leq \frac{1}{3} \bar{M}\left(\frac{c(x)}{k_{3}}\right)+\frac{k_{1}}{3 k_{3}} \bar{M} P^{-1} M\left(k_{2} k\right)+\frac{1}{3} M(\varphi) .
$$

Integrating this over $\Omega$, we get

$$
\int_{\Omega} \bar{M}\left[\frac{a\left(x, T_{k}\left(u_{p}\right), \frac{\varphi}{k_{4}}\right)}{3 k_{3}}\right] d x \leq C_{k}
$$

where $C_{k}$ is a constant depending on $k$.

We deduce that

$$
\int_{\Omega} a\left(x, T_{k}\left(u_{p}\right), \nabla T_{k}\left(u_{p}\right)\right) \varphi d x \leq C_{k} \forall \varphi \in\left(E_{M}(\Omega)\right)^{N},
$$

such that $\|\varphi\|_{M, \Omega}=1$, and so

$$
\left\|a\left(x, T_{k}\left(u_{p}\right), \nabla T_{k}\left(u_{p}\right)\right)\right\|_{\bar{M}, \Omega} \leq C_{k}
$$

which proves that $\left[a\left(x, T_{k}\left(u_{p}\right), \nabla T_{k}\left(u_{p}\right)\right)\right]_{p}$ is bounded in $\left(L_{\bar{M}}(\Omega)\right)^{N}$.

In a second step, we will demonstrate the almost everywhere convergence of the gradients, for that we consider the function $\varphi(t)=t e^{\sigma t^{2}}, \sigma>0$. It is known that when $\sigma>\left(\frac{b(k)}{2 \alpha}\right)^{2}$ one has

$$
\varphi^{\prime}(t)-\frac{b(k)}{\alpha}|\varphi(t)| \geq \frac{1}{2} \forall t \in \mathbb{R},
$$

where $k>0$ is a fixed real number which will be used as a level of the truncation, and we define the function $\rho_{m}$ where $m>k$, by

$$
\begin{cases}1 & \text { if } \quad|s| \leq m \\ m+1-|s| & \text { if } \quad m \leq|s| \leq m+1 \\ 0 & \text { if } \quad|s|>m+1\end{cases}
$$

For every fixed $k^{\prime}>0$, we have

$$
\begin{aligned}
\int_{\Omega} \frac{\left|\nabla u_{p}\right|^{p-2}}{h\left(u_{p}\right)^{p-1}} \nabla u_{p} & \nabla T_{k^{\prime}}\left(u_{p}-v\right) d x+\int_{\Omega} a\left(x, u_{p}, \nabla u_{p}\right) \nabla T_{k^{\prime}}\left(u_{p}-v\right) d x \\
& +\int_{\Omega} g\left(x, u_{p}, \nabla u_{p}\right) T_{k^{\prime}}\left(u_{p}-v\right) d x \leq \int_{\Omega} f T_{k^{\prime}}\left(u_{p}-v\right) d x
\end{aligned}
$$


choosing for all $\eta>0, v=T_{\eta}\left(u_{p}\right)-\varphi\left(T_{k}\left(u_{p}\right)-\theta u\right) \rho_{m}\left(u_{p}\right)$ in this inequality, where $\theta$ is a real number such that $0<\theta \leq 1$ then by letting $k^{\prime}$ and $\eta$ go to $+\infty$, we obtain

$$
\begin{aligned}
& \int_{\Omega} \frac{\left|\nabla u_{p}\right|^{p-2}}{h\left(u_{p}\right)^{p-1}} \nabla u_{p} \nabla \cdot\left[\varphi\left(T_{k}\left(u_{p}\right)-\theta u\right) \rho_{m}\left(u_{p}\right)\right] d x \\
& \quad+\int_{\Omega} a\left(x, u_{p}, \nabla u_{p}\right) \nabla \cdot\left[\varphi\left(T_{k}\left(u_{p}\right)-\theta u\right) \rho_{m}\left(u_{p}\right)\right] d x \\
& \quad+\int_{\Omega} g\left(x, u_{p}, \nabla u_{p}\right) \varphi\left(T_{k}\left(u_{p}\right)-\theta u\right) \rho_{m}\left(u_{p}\right) d x \leq \int_{\Omega} f \varphi\left(T_{k}\left(u_{p}\right)-\theta u\right) \rho_{m}\left(u_{p}\right) d x .
\end{aligned}
$$

We denote by $\epsilon_{i}(p, \theta)(i=0,1,2, \ldots)$ various sequences of real numbers which tend to 0 when $p$ tend to $+\infty$ and $\theta$ tend to 1 i.e.

$$
\lim _{\theta \rightarrow 1} \lim _{p \rightarrow+\infty} \epsilon_{i}(p, \theta)=0 .
$$

Since, we have $T_{k}\left(u_{p}\right) \longrightarrow u$, as $p \rightarrow \infty$, because $k \geq|u|$, hence

$$
\varphi\left(T_{k}\left(u_{p}\right)-\theta u\right) \rho_{m}\left(u_{p}\right) \rightarrow \varphi(u-\theta u) \rho_{m}(u) \text { weakly* in } L^{\infty}(\Omega),
$$

as $p \rightarrow+\infty$ and thus

$$
\int_{\Omega} f \varphi\left(T_{k}\left(u_{p}\right)-\theta u\right) \rho_{m}\left(u_{p}\right) d x \rightarrow \int_{\Omega} f \varphi(u-\theta u) \rho_{m}(u) d x,
$$

letting $\theta \rightarrow 1$, we obtain that

$$
\int_{\Omega} f \varphi(u-\theta u) \rho_{m}(u) d x \longrightarrow 0
$$

which means that

$$
\int_{\Omega} f \varphi\left(T_{k}\left(u_{p}\right)-\theta u\right) \rho_{m}\left(u_{p}\right) d x=\epsilon_{0}(p, \theta) .
$$

Denoting $\varphi\left(T_{k}\left(u_{p}\right)-\theta u\right) \rho_{m}\left(u_{p}\right)$ by $z_{p, m}^{\theta}$ and since $g\left(x, u_{p}, \nabla u_{p}\right) z_{p, m}^{\theta} \geq 0$ on the set $\{x \in \Omega$ : $\left.\left|u_{p}\right|>k\right\}$, so

$$
\left\langle A\left(u_{p}\right), z_{p, m}^{\theta}\right\rangle+\int_{\Omega} \frac{\left|\nabla u_{p}\right|^{p-2}}{h\left(u_{p}\right)^{p-1}} \nabla u_{p} \nabla z_{p, m}^{\theta} d x+\int_{\left\{\left|u_{p}\right| \leq k\right\}} g\left(x, u_{p}, \nabla u_{p}\right) z_{p, m}^{\theta} d x \leq \epsilon_{0}(p, \theta) .
$$

About the first term in this last inequality, we have

$$
\begin{aligned}
\left\langle A\left(u_{p}\right), z_{p, m}^{\theta}\right\rangle & =\int_{\Omega} a\left(x, u_{p}, \nabla u_{p}\right)\left[\nabla T_{k}\left(u_{p}\right)-\theta \nabla u\right] \varphi^{\prime}\left(T_{k}\left(u_{p}\right)-\theta u\right) \rho_{m}\left(u_{p}\right) d x \\
& +\int_{\Omega} a\left(x, u_{p}, \nabla u_{p}\right) \nabla u_{p} \varphi\left(T_{k}\left(u_{p}\right)-\theta u\right) \rho_{m}^{\prime}\left(u_{p}\right) d x \\
& =\int_{\left\{\left|u_{p}\right| \leq k\right\}} a\left(x, u_{p}, \nabla u_{p}\right)\left[\nabla T_{k}\left(u_{p}\right)-\theta \nabla u\right] \varphi^{\prime}\left(T_{k}\left(u_{p}\right)-\theta u\right) \rho_{m}\left(u_{p}\right) d x \\
& -\theta \int_{\left\{\left|u_{p}\right|>k\right\}} a\left(x, u_{p}, \nabla u_{p}\right) \nabla u \varphi^{\prime}\left(T_{k}\left(u_{p}\right)-\theta u\right) \rho_{m}\left(u_{p}\right) d x \\
& +\int_{\Omega} a\left(x, u_{p}, \nabla u_{p}\right) \nabla u_{p} \varphi\left(T_{k}\left(u_{p}\right)-\theta u\right) \rho_{m}^{\prime}\left(u_{p}\right) d x .
\end{aligned}
$$


Since $m>k$, we have $\rho_{m}\left(u_{p}\right)=1$ on the subset $\left\{x \in \Omega:\left|u_{p}\right| \leq k\right\}$, so

$$
\begin{aligned}
\left\langle A\left(u_{p}\right), z_{p, m}^{\theta}\right\rangle= & \int_{\left\{\left|u_{p}\right| \leq k\right\}} a\left(x, T_{k}\left(u_{p}\right), \nabla T_{k}\left(u_{p}\right)\right)\left[\nabla T_{k}\left(u_{p}\right)-\theta \nabla u\right] \varphi^{\prime}\left(T_{k}\left(u_{p}\right)-\theta u\right) d x \\
& -\theta \int_{\left\{\left|u_{p}\right|>k\right\}} a\left(x, u_{p}, \nabla u_{p}\right) \nabla u \varphi^{\prime}\left(T_{k}\left(u_{p}\right)-\theta u\right) \rho_{m}\left(u_{p}\right) d x \\
& +\int_{\Omega} a\left(x, u_{p}, \nabla u_{p}\right) \nabla u_{p} \varphi\left(T_{k}\left(u_{p}\right)-\theta u\right) \rho_{m}^{\prime}\left(u_{p}\right) d x,
\end{aligned}
$$

and then

$$
\begin{array}{r}
\left\langle A\left(u_{p}\right), z_{p, m}^{\theta}\right\rangle=\int_{\Omega}\left[a\left(x, T_{k}\left(u_{p}\right), \nabla T_{k}\left(u_{p}\right)\right)-a\left(x, T_{k}\left(u_{p}\right), \theta \nabla u\right)\right] \\
\quad \times\left[\nabla T_{k}\left(u_{p}\right)-\theta \nabla u\right] \varphi^{\prime}\left(T_{k}\left(u_{p}\right)-\theta u\right) d x \\
+\int_{\Omega} a\left(x, T_{k}\left(u_{p}\right), \theta \nabla u\right)\left[\nabla T_{k}\left(u_{p}\right)-\theta \nabla u\right] \varphi^{\prime}\left(T_{k}\left(u_{p}\right)-\theta u\right) d x \\
\quad-\theta \int_{\left\{\left|u_{p}\right|>k\right\}} a\left(x, u_{p}, \nabla u_{p}\right) \nabla u \varphi^{\prime}\left(T_{k}\left(u_{p}\right)-\theta u\right) \rho_{m}\left(u_{p}\right) d x \\
\quad+\int_{\Omega} a\left(x, u_{p}, \nabla u_{p}\right) \nabla u_{p} \varphi\left(T_{k}\left(u_{p}\right)-\theta u\right) \rho_{m}^{\prime}\left(u_{p}\right) d x .
\end{array}
$$

Thanks to Lemma 3, we have

$$
a\left(x, T_{k}\left(u_{p}\right), \theta \nabla u\right) \varphi^{\prime}\left(T_{k}\left(u_{p}\right)-\theta u\right) \longrightarrow a(x, u, \theta \nabla u) \varphi^{\prime}(u-\theta u),
$$

strongly in $\left(E_{\bar{M}}(\Omega)\right)^{N}$ as $p \rightarrow+\infty$, and from (3.15) we have

$$
\nabla T_{k}\left(u_{p}\right) \rightarrow \nabla u \text { weakly in }\left(L_{M}(\Omega)\right)^{N},
$$

we deduce that

$$
\begin{aligned}
& \int_{\Omega} a\left(x, T_{k}\left(u_{p}\right), \theta \nabla u\right)\left[\nabla T_{k}\left(u_{p}\right)-\theta \nabla u\right] \varphi^{\prime}\left(T_{k}\left(u_{p}\right)-\theta u\right) d x \\
& \quad \rightarrow \int_{\Omega} a(x, u, \theta \nabla u)[\nabla u-\theta \nabla u] \varphi^{\prime}(u-\theta u) d x \text { as } p \rightarrow+\infty,
\end{aligned}
$$

we pass to the limit when $\theta$ tends to 1 , we see that

$$
\int_{\Omega} a(x, u, \theta \nabla u)[\nabla u-\theta \nabla u] \varphi^{\prime}(u-\theta u) d x \rightarrow 0 \text { as } \theta \rightarrow 1,
$$

in other words, it means that

$$
\int_{\Omega} a\left(x, T_{k}\left(u_{p}\right), \theta \nabla u\right)\left[\nabla T_{k}\left(u_{p}\right)-\theta \nabla u\right] \varphi^{\prime}\left(T_{k}\left(u_{p}\right)-\theta u\right) d x=\epsilon_{1}(p, \theta) .
$$

Since $\rho_{m}\left(u_{p}\right)=0$ on the subset $\left\{\left|u_{p}\right|>m+1\right\}$, it follows therefore that

$$
\begin{aligned}
& -\theta \int_{\left\{\left|u_{p}\right|>k\right\}} a\left(x, u_{p}, \nabla u_{p}\right) \nabla u \varphi^{\prime}\left(T_{k}\left(u_{p}\right)-\theta u\right) \rho_{m}\left(u_{p}\right) d x \\
& =-\theta \int_{\left\{\left|u_{p}\right|>k\right\}} a\left(x, T_{m+1}\left(u_{p}\right), \nabla T_{m+1}\left(u_{p}\right)\right) \nabla u \varphi^{\prime}\left(T_{k}\left(u_{p}\right)-\theta u\right) \rho_{m}\left(u_{p}\right) d x .
\end{aligned}
$$


Furthermore, since $\left[a\left(x, T_{k}\left(u_{p}\right), \nabla T_{k}\left(u_{p}\right)\right)\right]_{p}$ is bounded in $\left(L_{\bar{M}}(\Omega)\right)^{N}$, so for a subsequence, we have

$$
a\left(x, T_{k}\left(u_{p}\right), \nabla T_{k}\left(u_{p}\right)\right) \rightarrow h_{k} \text { weakly in }\left(L_{\bar{M}}(\Omega)\right)^{N}
$$

for $\sigma\left(\Pi L_{\bar{M}}, \Pi E_{M}\right)$ for some $h_{k}$ in $\left(L_{\bar{M}}(\Omega)\right)^{N}$, and since

$$
\nabla u \in\left(E_{M}(\Omega)\right)^{N} \text {, }
$$

by letting $p \rightarrow+\infty$ and $\theta \rightarrow 1$, we obtain that

$$
\begin{aligned}
-\theta \int_{\left\{\left|u_{p}\right|>k\right\}} a & \left(x, T_{m+1}\left(u_{p}\right), \nabla T_{m+1}\left(u_{p}\right)\right) \nabla u \varphi^{\prime}\left(T_{k}\left(u_{p}\right)-\theta u\right) \rho_{m}\left(u_{p}\right) d x \\
& \rightarrow-\int_{\{|u|>k\}} h_{m+1} \nabla u \varphi^{\prime}(u-\theta u) \rho_{m}(u) d x .
\end{aligned}
$$

For $k>M_{0}$ (see (3.14)), we have

$$
\operatorname{meas}\{|u|>k\}=0 \text {. }
$$

Consequently,

which gives that

$$
\int_{\{|u|>k\}} h_{m+1} \nabla u \varphi^{\prime}(u-\theta u) \rho_{m}(u) d x=0,
$$

$$
-\theta \int_{\left\{\left|u_{p}\right|>k\right\}} a\left(x, u_{p}, \nabla u_{p}\right) \nabla u \varphi^{\prime}\left(T_{k}\left(u_{p}\right)-\theta u\right) \rho_{m}\left(u_{p}\right) d x=\epsilon_{2}(p, \theta) .
$$

On the other hand,

$$
\begin{aligned}
& \int_{\Omega} a\left(x, u_{p}, \nabla u_{p}\right) \nabla u_{p} \varphi\left(T_{k}\left(u_{p}\right)-\theta u\right) \rho_{m}^{\prime}\left(u_{p}\right) d x \\
& \quad=\int_{\left\{m \leq\left|u_{p}\right| \leq m+1\right\}} a\left(x, u_{p}, \nabla u_{p}\right) \nabla u_{p} \varphi\left(T_{k}\left(u_{p}\right)-\theta u\right) \rho_{m}^{\prime}\left(u_{p}\right) d x,
\end{aligned}
$$

hence for $k>M_{0}$, we get

$$
\begin{aligned}
\left|\int_{\Omega} a\left(x, u_{p}, \nabla u_{p}\right) \nabla u_{p} \varphi\left(T_{k}\left(u_{p}\right)-\theta u\right) \rho_{m}^{\prime}\left(u_{p}\right) d x\right| \\
\quad \leq \varphi(2 k) \int_{\left\{m \leq\left|u_{p}\right| \leq m+1\right\}} a\left(x, u_{p}, \nabla u_{p}\right) \nabla u_{p} d x .
\end{aligned}
$$

Taking $v=u_{p}-T_{1}\left(u_{p}-T_{m}\left(u_{p}\right)\right)$ in (3.9) and then letting $k \rightarrow \infty$, thus we have

$$
\begin{aligned}
& \int_{\Omega} \frac{\left|\nabla u_{p}\right|^{p-2}}{h\left(u_{p}\right)^{p-1}} \nabla u_{p} \nabla T_{1}\left(u_{p}-T_{m}\left(u_{p}\right)\right) d x+\int_{\Omega} a\left(x, u_{p}, \nabla u_{p}\right) \nabla T_{1}\left(u_{p}-T_{m}\left(u_{p}\right)\right) d x \\
& +\int_{\Omega} g\left(x, u_{p}, \nabla u_{p}\right) T_{1}\left(u_{p}-T_{m}\left(u_{p}\right)\right) d x \leq \int_{\Omega} f T_{1}\left(u_{p}-T_{m}\left(u_{p}\right)\right) d x
\end{aligned}
$$

then

$$
\int_{\left\{m \leq\left|u_{p}\right| \leq m+1\right\}} \frac{\left|\nabla u_{p}\right|^{p-2}}{h\left(u_{p}\right)^{p-1}} \nabla u_{p} . \nabla u_{p} d x+\int_{\left\{m \leq\left|u_{p}\right| \leq m+1\right\}} a\left(x, u_{p}, \nabla u_{p}\right) . \nabla u_{p} d x
$$




$$
+\int_{\Omega} g\left(x, u_{p}, \nabla u_{p}\right) T_{1}\left(u_{p}-T_{m}\left(u_{p}\right)\right) d x \leq \int_{\left\{\left|u_{p}\right| \geq m\right\}} f d x
$$

Since

$$
\int_{\left\{m \leq\left|u_{p}\right| \leq m+1\right\}} \frac{\left|\nabla u_{p}\right|^{p-2}}{h\left(u_{p}\right)^{p-1}} \nabla u_{p} . \nabla u_{p} d x \geq 0
$$

and

we obtain

$$
g\left(x, u_{p}, \nabla u_{p}\right) T_{1}\left(u_{p}-T_{m}\left(u_{p}\right)\right) \geq 0,
$$

$$
\int_{\left\{m \leq\left|u_{p}\right| \leq m+1\right\}} a\left(x, u_{p}, \nabla u_{p}\right) \nabla u_{p} d x \leq \int_{\left\{\left|u_{p}\right|>m\right\}} f d x,
$$

we deduce then that

$$
\left|\int_{\Omega} a\left(x, u_{p}, \nabla u_{p}\right) \nabla u_{p} \varphi\left(T_{k}\left(u_{p}\right)-\theta u\right) \rho_{m}^{\prime}\left(u_{p}\right) d x\right| \leq \varphi(2 k) \int_{\left\{\left|u_{p}\right|>m\right\}} f d x .
$$

Consequently,

$$
\begin{aligned}
\left\langle A\left(u_{p}\right), z_{p, m}^{\theta}\right\rangle \geq & -\varphi(2 k) \int_{\left\{\left|u_{p}\right|>m\right\}} f d x \\
+\int_{\Omega} & {\left[a\left(x, T_{k}\left(u_{p}\right), \nabla T_{k}\left(u_{p}\right)\right)-a\left(x, T_{k}\left(u_{p}\right), \theta \nabla u\right)\right] } \\
& \times\left[\nabla T_{k}\left(u_{p}\right)-\theta \nabla u\right] \varphi^{\prime}\left(T_{k}\left(u_{p}\right)-\theta u\right) d x+\epsilon_{3}(p, \theta) .
\end{aligned}
$$

Concerning the second term of (3.20), we can write it as follows

$$
\begin{array}{r}
\int_{\Omega} \frac{\left|\nabla u_{p}\right|^{p-2}}{h\left(u_{p}\right)^{p-1}} \nabla u_{p} \cdot\left[\nabla T_{k}\left(u_{p}\right)-\theta \nabla u\right] \varphi^{\prime}\left(T_{k}\left(u_{p}\right)-\theta u\right) \rho_{m}\left(u_{p}\right) d x \\
+\int_{\Omega} \frac{\left|\nabla u_{p}\right|^{p-2}}{h\left(u_{p}\right)^{p-1}} \nabla u_{p} . \nabla u_{p} \varphi\left(T_{k}\left(u_{p}\right)-\theta u\right) \rho_{m}^{\prime}\left(u_{p}\right) d x
\end{array}
$$

which is equal to

$$
\begin{aligned}
I:= & \int_{\Omega}\left[\frac{\left|\nabla u_{p}\right|^{p-2}}{h\left(u_{p}\right)^{p-1}} \nabla u_{p}-\frac{|\theta \nabla u|^{p-2}}{h\left(u_{p}\right)^{p-1}} \theta \nabla u\right]\left[\nabla T_{k}\left(u_{p}\right)-\theta \nabla u\right] \varphi^{\prime}\left(T_{k}\left(u_{p}\right)-\theta u\right) \rho_{m}\left(u_{p}\right) d x \\
& +\int_{\Omega} \theta^{p-1} \frac{|\nabla u|^{p-2}}{h\left(u_{p}\right)^{p-1}} \nabla u\left[\nabla T_{k}\left(u_{p}\right)-\theta \nabla u\right] \varphi^{\prime}\left(T_{k}\left(u_{p}\right)-\theta u\right) \rho_{m}\left(u_{p}\right) d x \\
& +\int_{\Omega} \frac{\left|\nabla u_{p}\right|^{p-2}}{h\left(u_{p}\right)^{p-1}} \nabla u_{p} \nabla u_{p} \varphi\left(T_{k}\left(u_{p}\right)-\theta u\right) \rho_{m}^{\prime}\left(u_{p}\right) d x
\end{aligned}
$$

by the definition of monotonicity, we have

$$
\int_{\Omega}\left[\frac{\left|\nabla u_{p}\right|^{p-2}}{h\left(u_{p}\right)^{p-1}} \nabla u_{p}-\frac{|\theta \nabla u|^{p-2}}{h\left(u_{p}\right)^{p-1}} \theta \nabla u\right]\left[\nabla T_{k}\left(u_{p}\right)-\theta \nabla u\right] \varphi^{\prime}\left(T_{k}\left(u_{p}\right)-\theta u\right) \rho_{m}\left(u_{p}\right) d x \geq 0,
$$


then

$$
\begin{gathered}
I \geq \int_{\Omega} \theta^{p-1} \frac{|\nabla u|^{p-2}}{h\left(u_{p}\right)^{p-1}} \nabla u \times\left[\nabla T_{k}\left(u_{p}\right)-\theta \nabla u\right] \varphi^{\prime}\left(T_{k}\left(u_{p}\right)-\theta u\right) \rho_{m}\left(u_{p}\right) d x \\
+\int_{\Omega} \frac{\left|\nabla u_{p}\right|^{p-2}}{h\left(u_{p}\right)^{p-1}} \nabla u_{p} \nabla u_{p} \varphi\left(T_{k}\left(u_{p}\right)-\theta u\right) \rho_{m}^{\prime}\left(u_{p}\right) d x .
\end{gathered}
$$

Since we have

$$
\begin{aligned}
& \left|\int_{\Omega} \theta^{p-1} \frac{|\nabla u|^{p-2}}{h\left(u_{p}\right)^{p-1}} \nabla u \times\left[\nabla T_{k}\left(u_{p}\right)-\theta \nabla u\right] \varphi^{\prime}\left(T_{k}\left(u_{p}\right)-\theta u\right) \rho_{m}\left(u_{p}\right) d x\right| \\
& \leq \int_{\Omega}\left(\theta \frac{|\nabla u|}{h\left(u_{p}\right)}\right)^{p-1}\left|\nabla T_{k}\left(u_{p}\right)-\theta \nabla u\right|\left|\varphi^{\prime}\left(T_{k}\left(u_{p}\right)-\theta u\right)\right|\left|\rho_{m}\left(u_{p}\right)\right| d x
\end{aligned}
$$

and then since $|\nabla u| \leq h(u)$, so

$$
\begin{aligned}
& \int_{\Omega}\left(\theta \frac{|\nabla u|}{h\left(u_{p}\right)}\right)^{p-1} \times\left[\nabla T_{k}\left(u_{p}\right)-\theta \nabla u\right]\left|\varphi^{\prime}\left(T_{k}\left(u_{p}\right)-\theta u\right)\right|\left|\rho_{m}\left(u_{p}\right)\right| d x \\
\leq & \int_{\Omega}\left(\theta \frac{h(u)}{h\left(u_{p}\right)}\right)^{p-1}\left|\nabla T_{k}\left(u_{p}\right)-\theta \nabla u\right|\left|\varphi^{\prime}\left(T_{k}\left(u_{p}\right)-\theta u\right)\right|\left|\rho_{m}\left(u_{p}\right)\right| d x
\end{aligned}
$$

and moreover since $\theta<1$ hence by passing the limit as $p \rightarrow+\infty$ and using the fact that $h\left(T_{m+1}\left(u_{p}\right)\right) \rightarrow h\left(T_{m+1}(u)\right)$ in $L^{\infty}(\Omega)$, we obtain that

$$
\int_{\Omega} \theta^{p-1} \frac{|\nabla u|^{p-2}}{h\left(u_{p}\right)^{p-1}} \nabla u \times\left[\nabla T_{k}\left(u_{p}\right)-\theta \nabla u\right] \varphi^{\prime}\left(T_{k}\left(u_{p}\right)-\theta u\right) \rho_{m}\left(u_{p}\right) d x=\epsilon_{4}(p, \theta) .
$$

On the other hand, we have for $k>M_{0}$

$$
\begin{aligned}
& \left|\int_{\Omega} \frac{\left|\nabla u_{p}\right|^{p-2}}{h\left(u_{p}\right)^{p-1}} \nabla u_{p} \nabla u_{p} \varphi\left(T_{k}\left(u_{p}\right)-\theta u\right) \rho_{m}^{\prime}\left(u_{p}\right) d x\right| \\
& \quad=\left|\int_{\left\{m \leq\left|u_{p}\right| \leq m+1\right\}} \frac{\left|\nabla u_{p}\right|^{p-2}}{h\left(u_{p}\right)^{p-1}} \nabla u_{p} \nabla u_{p} \varphi\left(T_{k}\left(u_{p}\right)-\theta u\right) \rho_{m}^{\prime}\left(u_{p}\right) d x\right| \\
& \quad \leq \varphi(2 k) \int_{\left\{m \leq\left|u_{p}\right| \leq m+1\right\}} \frac{\left|\nabla u_{p}\right|^{p}}{h\left(u_{p}\right)^{p-1}} d x .
\end{aligned}
$$

Going back to (3.22) and since

$$
g\left(x, u_{p}, \nabla u_{p}\right) T_{1}\left(u_{p}-T_{m}\left(u_{p}\right)\right) \geq 0,
$$

and

$$
\int_{\left\{m \leq\left|u_{p}\right| \leq m+1\right\}} a\left(x, u_{p}, \nabla u_{p}\right) \nabla u_{p} d x \geq \alpha \int_{\left\{m \leq\left|u_{p}\right| \leq m+1\right\}} M\left(\left|\nabla u_{p}\right|\right) d x \geq 0,
$$

it follows that

$$
\int_{\left\{m \leq\left|u_{p}\right| \leq m+1\right\}} \frac{\left|\nabla u_{p}\right|^{p}}{h\left(u_{p}\right)^{p-1}} d x \leq \int_{\left\{\left|u_{p}\right| \geq m\right\}} f d x
$$


which implies that

$$
\left|\int_{\Omega} \frac{\left|\nabla u_{p}\right|^{p-2}}{h\left(u_{p}\right)^{p-1}} \nabla u_{p} \nabla u_{p} \varphi\left(T_{k}\left(u_{p}\right)-\theta u\right) \rho_{m}^{\prime}\left(u_{p}\right) d x\right| \leq \varphi(2 k) \int_{\left\{\left|u_{p}\right| \geq m\right\}} f d x .
$$

For what concern the third term of (3.20), we have

$$
\begin{aligned}
& \left|\int_{\left\{\left|u_{p}\right| \leq k\right\}} g\left(x, u_{p}, \nabla u_{p}\right) z_{p, m}^{\theta} d x\right|=\left|\int_{\left\{\left|u_{p}\right| \leq k\right\}} g\left(x, T_{k}\left(u_{p}\right), \nabla T_{k}\left(u_{p}\right)\right) \varphi\left(T_{k}\left(u_{p}\right)-\theta u\right) d x\right| \\
& \leq \int_{\Omega} b(k) c^{\prime}(x)\left|\varphi\left(T_{k}\left(u_{p}\right)-\theta u\right)\right| d x+b(k) \int_{\Omega} M\left(\left|\nabla T_{k}\left(u_{p}\right)\right|\right)\left|\varphi\left(T_{k}\left(u_{p}\right)-\theta u\right)\right| d x \\
& \leq \epsilon_{5}(p, \theta)+\frac{b(k)}{\alpha} \int_{\Omega} a\left(x, T_{k}\left(u_{p}\right), \nabla T_{k}\left(u_{p}\right)\right) \nabla T_{k}\left(u_{p}\right)\left|\varphi\left(T_{k}\left(u_{p}\right)-\theta u\right)\right| d x .
\end{aligned}
$$

Moreover, since

$$
\begin{aligned}
& \frac{b(k)}{\alpha} \int_{\Omega} a\left(x, T_{k}\left(u_{p}\right), \nabla T_{k}\left(u_{p}\right)\right) \nabla T_{k}\left(u_{p}\right)\left|\varphi\left(T_{k}\left(u_{p}\right)-\theta u\right)\right| d x \\
& =\frac{b(k)}{\alpha} \int_{\Omega}\left[a\left(x, T_{k}\left(u_{p}\right), \nabla T_{k}\left(u_{p}\right)\right)-a\left(x, T_{k}\left(u_{p}\right), \theta \nabla u\right)\right] \\
& \times\left[\nabla T_{k}\left(u_{p}\right)-\theta \nabla u\right]\left|\varphi\left(T_{k}\left(u_{p}\right)-\theta u\right)\right| d x \\
& +\frac{b(k)}{\alpha} \int_{\Omega} a\left(x, T_{k}\left(u_{p}\right), \theta \nabla u\right)\left[\nabla T_{k}\left(u_{p}\right)-\theta \nabla u\right]\left|\varphi\left(T_{k}\left(u_{p}\right)-\theta u\right)\right| d x \\
& -\frac{b(k)}{\alpha} \theta \int_{\Omega} a\left(x, T_{k}\left(u_{p}\right), \nabla T_{k}\left(u_{p}\right)\right) \nabla u\left|\varphi\left(T_{k}\left(u_{p}\right)-\theta u\right)\right| d x,
\end{aligned}
$$

and in the same way as the terms above, we remark that

$$
\left.\frac{b(k)}{\alpha} \int_{\Omega} a\left(x, T_{k}\left(u_{p}\right), \theta \nabla u\right)\left[\nabla T_{k}\left(u_{p}\right)-\theta \nabla u\right)\right]\left|\varphi\left(T_{k}\left(u_{p}\right)-\theta u\right)\right| d x=\epsilon_{6}(p, \theta)
$$

and that

$$
-\frac{b(k)}{\alpha} \theta \int_{\Omega} a\left(x, T_{k}\left(u_{p}\right), \nabla T_{k}\left(u_{p}\right)\right) \nabla u\left|\varphi\left(T_{k}\left(u_{p}\right)-\theta u\right)\right| d x=\epsilon_{7}(p, \theta),
$$

then we conclude that

$$
\begin{aligned}
\left|\int_{\left\{\left|u_{p}\right| \leq k\right\}} g\left(x, u_{p}, \nabla u_{p}\right) z_{p, m}^{\theta} d x\right| & \\
\leq \frac{b(k)}{\alpha} \int_{\Omega} & {\left[a\left(, T_{k}\left(u_{p}\right), \nabla T_{k}\left(u_{p}\right)\right)-a\left(x, T_{k}\left(u_{p}\right), \theta \nabla u\right)\right] } \\
& \times\left[\nabla T_{k}\left(u_{p}\right)-\theta \nabla u\right]\left|\varphi\left(T_{k}\left(u_{p}\right)-\theta u\right)\right| d x+\epsilon_{8}(p, \theta) .
\end{aligned}
$$

Combining the estimates (3.23), (3.24), (3.25) and (3.26), we obtain

$$
-2 \varphi(2 k) \int_{\left\{\left|u_{p}\right|>m\right\}} f d x
$$


NONLINEAR INEQUALITIES WITH GRADIENT CONSTRAINTS IN ORLICZ SPACES

$$
\begin{aligned}
+\int_{\Omega}\left[a\left(x, T_{k}\left(u_{p}\right), \nabla T_{k}\left(u_{p}\right)\right)-\right. & \left.a\left(x, T_{k}\left(u_{p}\right), \theta \nabla u\right)\right] \\
& \times\left[\nabla T_{k}\left(u_{p}\right)-\theta \nabla u\right] \varphi^{\prime}\left(T_{k}\left(u_{p}\right)-\theta u\right) d x \\
\leq \frac{b(k)}{\alpha} \int_{\Omega}\left[a \left(x, T_{k}\left(u_{p}\right),\right.\right. & \left.\left.\nabla T_{k}\left(u_{p}\right)\right)-a\left(x, T_{k}\left(u_{p}\right), \theta \nabla u\right)\right] \\
& \times\left[\nabla T_{k}\left(u_{p}\right)-\theta \nabla u\right]\left|\varphi\left(T_{k}\left(u_{p}\right)-\theta u\right)\right| d x+\epsilon_{9}(p, \theta)
\end{aligned}
$$

so

$$
\begin{aligned}
& \int_{\Omega}\left[a\left(x, T_{k}\left(u_{p}\right), \nabla T_{k}\left(u_{p}\right)\right)-a\left(x, T_{k}\left(u_{p}\right), \theta \nabla u\right)\right] \\
& \quad \times\left[\nabla T_{k}\left(u_{p}\right)-\theta \nabla u\right]\left[\varphi^{\prime}\left(T_{k}\left(u_{p}\right)-\theta u\right)-\frac{b(k)}{\alpha}\left|\varphi\left(T_{k}\left(u_{p}\right)-\theta u\right)\right|\right] d x \\
& \quad \leq 2 \varphi(2 k) \int_{\left\{\left|u_{p}\right| \geq m\right\}} f d x+\epsilon_{9}(p, \theta) .
\end{aligned}
$$

Consequently, from (3.17), we have

$$
\begin{aligned}
\int_{\Omega}\left[a\left(x, T_{k}\left(u_{p}\right), \nabla T_{k}\left(u_{p}\right)\right)\right. & \left.-a\left(x, T_{k}\left(u_{p}\right), \theta \nabla u\right)\right]\left[\nabla T_{k}\left(u_{p}\right)-\theta \nabla u\right] d x \\
& \leq 4 \varphi(2 k) \int_{\left\{\left|u_{p}\right| \geq m\right\}} f d x+2 \epsilon_{9}(p, \theta) .
\end{aligned}
$$

On the other hand,

$$
\begin{aligned}
& \int_{\Omega}\left[a\left(x, T_{k}\left(u_{p}\right), \nabla T_{k}\left(u_{p}\right)\right)-a\left(x, T_{k}\left(u_{p}\right), \nabla u\right)\right] \times\left[\nabla T_{k}\left(u_{p}\right)-\nabla u\right] d x \\
= & \int_{\Omega}\left[a\left(x, T_{k}\left(u_{p}\right), \nabla T_{k}\left(u_{p}\right)\right)-a\left(x, T_{k}\left(u_{p}\right), \theta \nabla u\right)\right] \times\left[\nabla T_{k}\left(u_{p}\right)-\theta \nabla u\right] d x \\
& +\int_{\Omega} a\left(x, T_{k}\left(u_{p}\right), \nabla T_{k}\left(u_{p}\right)\right)[\theta \nabla u-\nabla u] d x \\
& -\int_{\Omega} a\left(x, T_{k}\left(u_{p}\right), \nabla u\right)\left[\nabla T_{k}\left(u_{p}\right)-\nabla u\right] d x \\
& +\int_{\Omega} a\left(x, T_{k}\left(u_{p}\right), \theta \nabla u\right)\left[\nabla T_{k}\left(u_{p}\right)-\theta \nabla u\right] d x .
\end{aligned}
$$

Letting $p \rightarrow+\infty$ and $\theta \rightarrow 1$ in the last three terms of the last side of the above equality, we obtain that

$$
\int_{\Omega} a\left(x, T_{k}\left(u_{p}\right), \nabla T_{k}\left(u_{p}\right)\right)[\theta \nabla u-\nabla u] d x=\epsilon_{10}(p, \theta)
$$

and

$$
\int_{\Omega} a\left(x, T_{k}\left(u_{p}\right), \nabla u\right)\left[\nabla T_{k}\left(u_{p}\right)-\nabla u\right] d x=\epsilon_{11}(p, \theta)
$$

and

$$
\int_{\Omega} a\left(x, T_{k}\left(u_{p}\right), \theta \nabla u\right)\left[\nabla T_{k}\left(u_{p}\right)-\theta \nabla u\right] d x=\epsilon_{12}(p, \theta)
$$


which implies that

$$
\begin{aligned}
& \int_{\Omega}\left[a\left(x, T_{k}\left(u_{p}\right), \nabla T_{k}\left(u_{p}\right)\right)-a\left(x, T_{k}\left(u_{p}\right), \nabla u\right)\right]\left[\nabla T_{k}\left(u_{p}\right)-\nabla u\right] d x \\
= & \int_{\Omega}\left[a\left(x, T_{k}\left(u_{p}\right), \nabla T_{k}\left(u_{p}\right)\right)-a\left(x, T_{k}\left(u_{p}\right), \theta \nabla u\right)\right]\left[\nabla T_{k}\left(u_{p}\right)-\theta \nabla u\right] d x+\epsilon_{13}(p, \theta) \\
\leq & \epsilon_{14}(p, \theta)+4 \varphi(2 k) \int_{\left\{\left|u_{p}\right| \geq m\right\}} f d x,
\end{aligned}
$$

we passing to the limit sup over $p$ and then over $\theta$, we get

$$
\begin{aligned}
& \underset{p \rightarrow+\infty}{\limsup } \int_{\Omega}\left[a\left(x, T_{k}\left(u_{p}\right), \nabla T_{k}\left(u_{p}\right)\right)-a\left(x, T_{k}\left(u_{p}\right), \nabla u\right)\right]\left[\nabla T_{k}\left(u_{p}\right)-\nabla u\right] d x \\
& \quad \leq 4 \varphi(2 k) \int_{\{|u| \geq m\}} f d x,
\end{aligned}
$$

by letting $m \rightarrow \infty$ and due to the fact that $|\{|u| \geq m\}| \rightarrow 0$, we obtain that

$$
\int_{\Omega}\left[a\left(x, T_{k}\left(u_{p}\right), \nabla T_{k}\left(u_{p}\right)\right)-a\left(x, T_{k}\left(u_{p}\right), \nabla u\right)\right] \times\left[\nabla T_{k}\left(u_{p}\right)-\nabla u\right] d x \rightarrow 0
$$

as $p \rightarrow \infty$.

We deduce then as in [3] that there exists a subsequence still denoted by $u_{p}$ such that

$$
\nabla u_{p} \rightarrow \nabla u \text { a.e. in } \Omega \text {, }
$$

which gives that, for all $k>0$,

$$
\begin{aligned}
a\left(x, T_{k}\left(u_{p}\right), \nabla T_{k}\left(u_{p}\right)\right) \rightarrow & a\left(x, T_{k}(u), \nabla u\right) \text { weakly in }\left(L_{\bar{M}}(\Omega)\right)^{N} \\
& \text { for } \sigma\left(\Pi L_{\bar{M}}(\Omega), \Pi E_{M}(\Omega)\right) .
\end{aligned}
$$

After showing the almost everywhere convergence of the gradients, in the third step we will prove the modular convergence of the truncations.

Returning to (3.27), we can write

$$
\begin{aligned}
& \int_{\Omega} a\left(x, T_{k}\left(u_{p}\right), \nabla T_{k}\left(u_{p}\right)\right) \nabla T_{k}\left(u_{p}\right) d x \\
& \leq \int_{\Omega} a\left(x, T_{k}\left(u_{p}\right), \nabla T_{k}\left(u_{p}\right)\right) \theta \nabla u d x \\
& +\int_{\Omega} a\left(x, T_{k}\left(u_{p}\right), \theta \nabla u\right) \times\left[\nabla T_{k}\left(u_{p}\right)-\theta \nabla u\right] d x \\
& +2 \epsilon_{9}(p, \theta)+4 \varphi(2 k) \int_{\left\{\left|u_{p}\right| \geq m\right\}} f d x,
\end{aligned}
$$

by using (3.28), this implies that

$$
\begin{aligned}
& \int_{\Omega} a\left(x, T_{k}\left(u_{p}\right), \nabla T_{k}\left(u_{p}\right)\right) \nabla T_{k}\left(u_{p}\right) d x \\
& \quad \leq \int_{\Omega} a\left(x, T_{k}\left(u_{p}\right), \nabla T_{k}\left(u_{p}\right)\right) \theta \nabla u d x+\epsilon_{15}(p, \theta)+4 \varphi(2 k) \int_{\left\{\left|u_{p}\right| \geq m\right\}} f d x,
\end{aligned}
$$


passing to the limit sup over $p$, we have

$$
\begin{aligned}
\limsup _{p} & \int_{\Omega} a\left(x, T_{k}\left(u_{p}\right), \nabla T_{k}\left(u_{p}\right)\right) \nabla T_{k}\left(u_{p}\right) d x \\
& \leq \int_{\Omega} a(x, u, \nabla u) \theta \nabla u d x+\lim _{p \rightarrow+\infty} \epsilon_{15}(p, \theta)+4 \varphi(2 k) \int_{\{|u| \geq m\}} f d x
\end{aligned}
$$

letting $\theta \rightarrow 1$, we get

$$
\begin{aligned}
\lim \sup _{p} & \int_{\Omega} a\left(x, T_{k}\left(u_{p}\right), \nabla T_{k}\left(u_{p}\right)\right) \nabla T_{k}\left(u_{p}\right) d x \\
& \leq \int_{\Omega} a(x, u, \nabla u) \nabla u d x+4 \varphi(2 k) \int_{\{|u| \geq m\}} f d x
\end{aligned}
$$

letting $m \rightarrow+\infty$, we obtain

$$
\limsup _{p} \int_{\Omega} a\left(x, T_{k}\left(u_{p}\right), \nabla T_{k}\left(u_{p}\right)\right) \nabla T_{k}\left(u_{p}\right) d x \leq \int_{\Omega} a(x, u, \nabla u) \nabla u d x .
$$

On the other hand, by using the Lemma's Fatou

$$
\int_{\Omega} a(x, u, \nabla u) \nabla u d x \leq \liminf _{p \rightarrow+\infty} \int_{\Omega} a\left(x, T_{k}\left(u_{p}\right), \nabla T_{k}\left(u_{p}\right)\right) \nabla T_{k}\left(u_{p}\right) d x,
$$

so

$$
\int_{\Omega} a\left(x, T_{k}\left(u_{p}\right), \nabla T_{k}\left(u_{p}\right)\right) \nabla T_{k}\left(u_{p}\right) d x \rightarrow \int_{\Omega} a(x, u, \nabla u) \nabla u d x \text {, as } p \rightarrow+\infty
$$

and, by using Lemma 4, we conclude that

$$
a\left(x, T_{k}\left(u_{p}\right), \nabla T_{k}\left(u_{p}\right)\right) \nabla T_{k}\left(u_{p}\right) \rightarrow a(x, u, \nabla u) \nabla u, \quad \text { in } L^{1}(\Omega) .
$$

This implies, by using (3.3), that

$$
T_{k}\left(u_{p}\right) \rightarrow u \text { in } W_{0}^{1} L_{M}(\Omega)
$$

for the modular convergence for all $k>\|u\|_{\infty}$.

Now we will show the equi-integrability of the non-linearities. In fact, what we want to demonstrate is that

$$
g\left(x, u_{p}, \nabla u_{p}\right) \rightarrow g(x, u, \nabla u) \text { strongly in } L^{1}(\Omega)
$$

by using Vitali's theorem. Since

$$
\nabla u_{p} \rightarrow \nabla u \text { a.e in } \Omega,
$$

then

$$
g\left(x, u_{p}, \nabla u_{p}\right) \rightarrow g(x, u, \nabla u) \text { a.e in } \Omega,
$$

it suffices to prove that $g\left(x, u_{p}, \nabla u_{p}\right)$ are uniformaly equi-integrable in $\Omega$.

Let $E \subset \Omega$ be a measurable subset of $\Omega$. For any $m>0$, we have

$$
\int_{E} g\left(x, u_{p}, \nabla u_{p}\right) d x=\int_{E \cap\left\{\left|u_{p}\right| \leq m\right\}} g\left(x, u_{p}, \nabla u_{p}\right) d x+\int_{\left\{\left|u_{p}\right|>m\right\}} g\left(x, u_{p}, \nabla u_{p}\right) d x
$$


Taking $v=T_{m}\left(u_{p}\right)$ in (3.9), then we get

$$
\int_{\left\{\left|u_{p}\right|>m\right\}} g\left(x, u_{p}, \nabla u_{p}\right) d x \leq \int_{\left\{\left|u_{p}\right| \geq m\right\}} f d x .
$$

Let $\epsilon>0$. So there exists $m=m(\epsilon)>0$ such that

$$
\int_{\left\{\left|u_{p}\right|>m\right\}} g\left(x, u_{p}, \nabla u_{p}\right) d x \leq \frac{\epsilon}{2} \text {. }
$$

On the other hand, we have

$$
\begin{aligned}
\int_{E \cap\left\{\left|u_{p}\right| \leq m\right\}} g\left(x, u_{p}, \nabla u_{p}\right) d x & \leq \int_{E} g\left(x, T_{m}\left(u_{p}\right), \nabla T_{m}\left(u_{p}\right)\right) d x \\
& \leq b(m) \int_{E}\left[c^{\prime}(x)+M\left(\left|\nabla T_{m}\left(u_{p}\right)\right|\right)\right] d x \\
& \leq b(m) \int_{E} a\left(x, T_{m}\left(u_{p}\right), \nabla T_{m}\left(u_{p}\right)\right) \nabla T_{m}\left(u_{p}\right) d x \\
& +b(m) \int_{E} c^{\prime}(x) d x .
\end{aligned}
$$

From (3.31) and since $c^{\prime}(x) \in L^{1}(\Omega)$, there exists $\mu>0$ such that

$$
E<\mu \Longrightarrow \int_{E \cap\left\{\left|u_{p}\right| \leq m\right\}} g\left(x, u_{p}, \nabla u_{p}\right) d x \leq \frac{\epsilon}{2} .
$$

Consequently,

$$
E<\mu \Longrightarrow \int_{E} g\left(x, u_{p}, \nabla u_{p}\right) d x \leq \epsilon,
$$

which proves that $g\left(x, u_{p}, \nabla u_{p}\right)$ are uniformly equi-integrable in $\Omega$ as required.

Finally, we finish our proof by the passage to the limit.

Let $\varphi$ be a function such that $|\nabla \varphi| \leq h(u)$, choosing $v=\theta \varphi$ in (3.9), where $0<\theta \leq 1$ is a real number, we have for every fixed $k>0$ that

$$
\begin{aligned}
& \int_{\Omega} \frac{\left|\nabla u_{p}\right|^{p-2}}{h\left(u_{p}\right)^{p-1}} \nabla u_{p} \nabla T_{k}\left(u_{p}-\theta \varphi\right) d x+\int_{\Omega} a\left(x, u_{p}, \nabla u_{p}\right) \nabla T_{k}\left(u_{p}-\theta \varphi\right) d x \\
& \quad+\int_{\Omega} g\left(x, u_{p}, \nabla u_{p}\right) T_{k}\left(u_{p}-\theta \varphi\right) d x \leq \int_{\Omega} f T_{k}\left(u_{p}-\theta \varphi\right) d x .
\end{aligned}
$$

On the other hand, we have

$$
\begin{aligned}
& \int_{\Omega} \frac{\left|\nabla u_{p}\right|^{p-2}}{h\left(u_{p}\right)^{p-1}} \nabla u_{p}\left[\nabla T_{k}\left(u_{p}\right)-\theta \nabla \varphi\right] d x \\
& =\int_{\Omega}\left[\frac{\left|\nabla u_{p}\right|^{p-2}}{h\left(u_{p}\right)^{p-1}} \nabla u_{p}-\theta^{p-1} \frac{|\nabla \varphi|^{p-2}}{h\left(u_{p}\right)^{p-1}} \nabla \varphi\right]\left[\nabla T_{k}\left(u_{p}\right)-\theta \nabla \varphi\right] d x \\
& +\int_{\Omega} \theta^{p-1} \frac{|\nabla \varphi|^{p-2}}{h\left(u_{p}\right)^{p-1}} \nabla \varphi\left[\nabla T_{k}\left(u_{p}\right)-\theta \nabla \varphi\right] d x
\end{aligned}
$$




$$
\geq \int_{\Omega} \theta^{p-1} \frac{|\nabla \varphi|^{p-2}}{h\left(u_{p}\right)^{p-1}} \nabla \varphi\left[\nabla T_{k}\left(u_{p}\right)-\theta \nabla \varphi\right] d x
$$

Since $|\nabla \varphi| \leq h(u)$, so

$$
\left|\int_{\Omega} \theta^{p-1} \frac{|\nabla \varphi|^{p-2}}{h\left(u_{p}\right)^{p-1}} \nabla \varphi\left[\nabla T_{k}\left(u_{p}\right)-\theta \nabla \varphi\right] d x\right| \leq \int_{\Omega}\left(\theta \frac{h(u)}{h\left(u_{p}\right)}\right)^{p-1}\left|\nabla T_{k}\left(u_{p}\right)-\theta \nabla \varphi\right| d x,
$$

and as $p \rightarrow+\infty$ and then $\theta \rightarrow 1$, we have

$$
\int_{\Omega}\left(\theta \frac{h(u)}{h\left(u_{p}\right)}\right)^{p-1}\left|\nabla T_{k}\left(u_{p}\right)-\theta \nabla \varphi\right| d x \rightarrow 0,
$$

hence

$$
\lim _{\theta \rightarrow 1} \lim _{p \rightarrow+\infty} \int_{\Omega} \frac{\left|\nabla u_{p}\right|^{p-2}}{h\left(u_{p}\right)^{p-1}} \nabla u_{p}\left[\nabla T_{k}\left(u_{p}\right)-\theta \nabla \varphi\right] d x \geq 0 .
$$

We pass to the limit over $p$ on the other integrals in (3.32) and then over $\theta$ and lastly over $k$, consequently we have

$$
\int_{\Omega} a(x, u, \nabla u)[\nabla u-\nabla \varphi] d x+\int_{\Omega} g(x, u, \nabla u)(u-\varphi) d x \leq \int_{\Omega} f(u-\varphi) d x .
$$

for all $\varphi \in W_{0}^{1, \infty}(\Omega)$ such that $|\nabla \varphi| \leq h(u)$.

\section{References}

[1] R. Adams, Sobolev spaces, Pure and Applied Mathematics, 65. Academic Press [A subsidiary of Harcourt Brace Jovanovich, Publishers], New York-London, 1975.

[2] A. Atlas, F. Karami and D.Meskine, The Perona-Malik inequality and application to image denoising, Nonlinear Anal. Real World Appl. 18 (2014), 57-68.

[3] A.Benkirane And A. Elmahi, Almost everywhere convergence of the gradients of solutions to elliptic equations in Orlicz spaces and application, Nonlinear Anal. 2811 (1997), 1769-1784.

[4] A. Elmahi And D. Meskine, Non-linear elliptic problems having natural growth and $L^{1}$ data in Orlicz spaces, Ann. Mat. Pura Appl. 1842 (2005), 161-184.

[5] A. Elmahi and D. Meskine, Existence of solutions for elliptic equations having natural growth terms in Orlicz spaces, Abstr. Appl. Anal. 12 (2004), 1031-1045.

[6] J.-P. Gossez, Nonlinear elliptic boundary value problems for equations with rapidly (or slowly) increasing coefficients, Trans. Amer. Math. Soc. 190 (1974), 163-205.

[7] J.-P. Gossez, Some approximation properties in Orlicz-Sobolev spaces, Studia Math. 741 (1982), 17-24.

[8] J.-P. Gossez, A strongly nonlinear elliptic problem in Orlicz-Sobolev spaces, Nonlinear functional analysis and its applications, Part 1 (Berkeley, Calif., 1983), Proc. Sympos. Pure Math., 45, Part 1, Amer. Math. Soc., Providence, RI, (1986), 455-462.

[9] A. Kufner, O. John and S. Fucik, Function spaces, Monographs and Textbooks on Mechanics of Solids and Fluids; Mechanics: Analysis. Noordhoff International Publishing, Leyden; Academia, Prague, 1977.

[10] M. Krasnosel'skit and Ya. RutickiI, Convex functions and Orlicz spaces, Translated from the first Russian edition by Leo F. Boron. P. Noordhoff Ltd., Groningen 1961.

[11] Miranda, Fernando; Rodrigues, Jos Francisco; Santos, Lisa., Evolutionary quasi-variational and variational inequalities with constraints on the derivatives, Adv. Nonlinear Anal. 9 (2020), no. 1, 250-277.

[12] L. PRIGOzHIN, Variational model of sandpile growth, European J. Appl. Math.7 3 (1996), 225-235. 
[13] J.-F. Rodrigues And L. Santos, A parabolic quasi-variational inequality arising in a superconductivity model, Ann. Scuola Norm. Sup. Pisa Cl. Sci. (4) 291 (2000), 153-169. 